\title{
ISOTROPIZATION OF HOMOGENEOUS COSMOLOGICAL MODELS
}

\author{
I. D. NOVIKOV \\ Institute of Applied Mathematics, USSR Academy of Sciences, Moscow, U.S.S.R.
}

\begin{abstract}
Observations primarily of the microwave background radiation show that the Universe expands isotropically with a high degree of accuracy at the present time and that the matter distribution is homogeneous on a large scale. Thus, the Friedmann cosmological models are a good approximation today for the expanding Universe. This is valid for at least some period of time in the past too. But how did the Universe expand and what was the matter distribution close to the starting point, near the cosmological singularity?
\end{abstract}

Recently the possibility of non-Friedmannian initial conditions with a subsequent cosmological expansion which approximates very closely to the Friedmann model has been intensively discussed. Such an expanding Universe approximates to the Friedmann model with a certain degree of accuracy. However, in the case of a nonFriedmannian beginning, small deviations from the Friedmann model should be present today in the Universe. If it were possible to observe such deviations at the present day, one could in principle reconstruct the entire pattern of expansion near the singularity.

This problem in its general form is of extreme theoretical difficulty. Only the idealized cases of anisotropic but homogeneous cosmological models have been investigated adequately. These particular cases were chosen first, of course, due to their relative mathematical simplicity. But it should be emphasized that near the singularity these models differ in important ways from the Friedmann models, and thus enable us to understand what might happen in the past in a much wider class of models than the Friedmann cases. The anisotropic homogeneous models enable us to treat directed mass flows, fluxes of particles and the presence of a homogeneous cosmological magnetic field, i.e. to investigate processes which are impossible in the Friedmann model and which might be of importance in the past. Furthermore, the results of Belinsky et al. (1972) show that the expansion pattern near the singularity in the most general inhomogeneous solution coincides locally with that in the homogeneous case. This conclusion is valid until the scale of the inhomogeneity greatly exceeds the radius of the horizon - the light path from the instant of the singularity.

Henceforth in this lecture, only anisotropic homogeneous models will be considered.

The expansion of these models near the singularity in general is anisotropic. This stage was discussed in the report by Belinsky et al. How do these models expand? Do they approach the Friedmann model? It has usually been assumed that models from a rather wide class do indeed approach the Friedmann model as they expand. Some particular models of that kind have been investigated. In the present report the problem is treated in general form. 
First of all we recall some well-known results. Schucking and Heckmann (1958) and Heckmann and Schucking (1962) showed that anisotropically expanding models with isotropic spatial curvature (models of Bianchi types I and V) tend to an isotropic expansion as time passes. Their evolution may be divided into two stages. The first stage is an essentially anisotropic expansion which can be described by the Kasner solution:

$$
\begin{aligned}
& \mathrm{d} s^{2}=c^{2} \mathrm{~d} t^{2}-t^{2 p_{1}} \mathrm{~d} x^{1^{2}}-t^{2 p_{2}} \mathrm{~d} x^{2^{2}}-t^{2 p_{3}} \mathrm{~d} x^{3^{2}} \\
& p_{i}=\text { const } ; \quad p_{1}+p_{2}+p_{3}=p_{1}^{2}+p_{2}^{2}+p_{3}^{2}=1 .
\end{aligned}
$$

The 'Hubble constants' in the three perpendicular directions are different $H_{1} \neq$ $\neq H_{2} \neq H_{3}$, and one of them has a negative value. In Figure $1 \Delta H_{i} / H$ - the deviation of $H_{i}$ from the average value $H=\frac{1}{3}\left(H_{1}+H_{2}+H_{3}\right)$ - is shown; $\Delta H_{i}=H_{i}-H$. At this stage the stress-energy terms in the Einstein equations are negligible and for this reason it is called the 'vacuum' stage.

In the next stage, when the stress-energy terms in the Einstein equations become important, the solution rapidly approaches the Friedmann case. The attenuation law of $\Delta H_{i} / H$ for the equation of state $p=\varepsilon / 3$ is

$$
\Delta H_{i} / H \approx\left(t_{\mathrm{F}} / t\right)^{1 / 2} \text {. }
$$

The moment $t_{\mathrm{F}}$ at which the solution becomes Friedmannian is an arbitrary parameter of the model.

These first examples have demonstrated the possibility of an extremely anisotropic beginning to the expansion with subsequent rapid isotropization.

In a series of papers by Doroshkevich, Zel'dovich and Novikov and by Misner

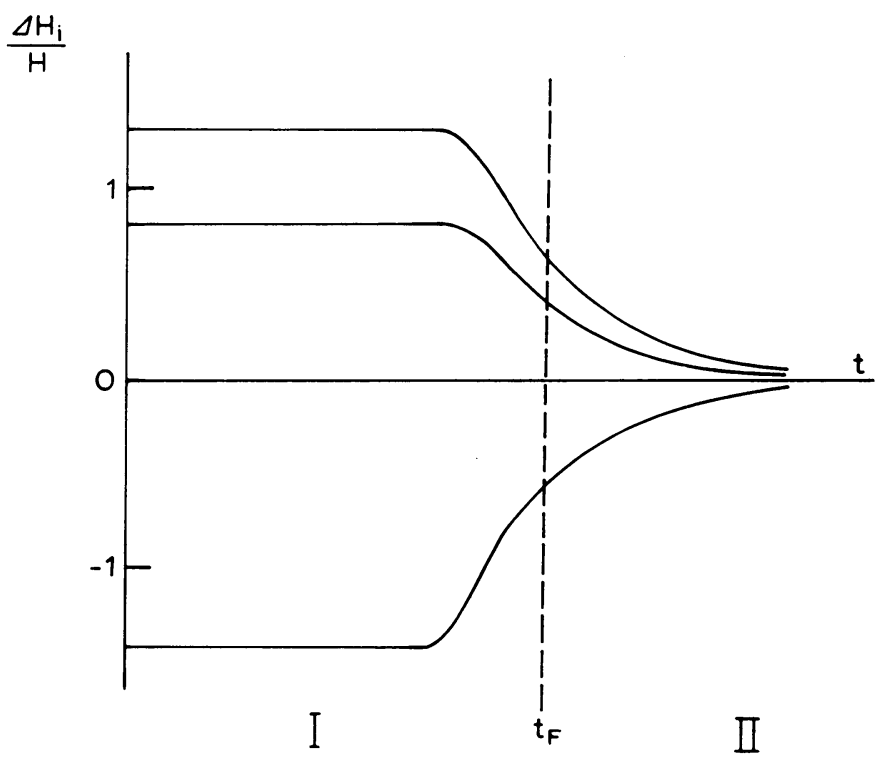

Fig. 1. The behaviour of $A H_{i} / H$ during the expansion of the anisotropic cosmological models with isotropic three-spaces. 
and his co-workers (see the survey by Doroshkevich et al., 1969) it was shown that a directed flux of weakly interacting particles (gravitons, neutrinos) arises due to the anisotropic beginning of the expansion. The flux flows along the axis of negative $p_{i}$. Novikov (1970) has shown that a directed flow of matter as a whole with respect to a homogeneous reference frame should arise along the same axis. This flow is due to the growth of a small peculiar velocity. Both fluxes (of particles and of matter) grow during the vacuum stage. Their growth causes the terms depending on the energy of these fluxes in the Einstein equations to become significant. Through these terms the expansion readjusts itself in such a way as to extinguish these fluxes (see the survey by Zel'dovich and Novikov, 1974). Here again the tendency to automatic isotropization is apparent.

All these examples give us reason to believe that an anisotropic homogeneous initial stage of expansion is possible, and as the expansion proceeds it tends to become isotropic.

But the problem has turned out to be a bit more complicated.

Our further discussion is based upon results obtained by Doroshkevich et al. (1973a, b) and Lukash (1973).

Let us consider the general case of the expansion of homogeneous models in which not only the initial expansion near the singularity is anisotropic but the spatial curvature is also anisotropic. For comparison of the theoretical results with the observational data the most interesting cases are the models of Bianchi types VII and IX, and only those will be discussed here. The model of type VII has an infinite threespace and may approximate in the course of expansion to the open Friedmann model. The model of type IX has a closed three-space and may approximate at some stage to the closed Friedmann model.

First we assume that no directed flux of particles or of matter as a whole is present. The first 'vacuum' stage of expansion is essentially anisotropic. In general it has the oscillatory character described in the reports by Belinsky et al. and by Misner. The variations in $\Delta H_{i}$ for this stage are shown in Figure 2. It is the anisotropy of the curvature which causes the solution to oscillate. Note that the volume increases monotonically.

Then the second stage commences. It begins when the stress-energy terms influence significantly the solution of the Einstein equations. The influence of these terms results in the isotropization of the expansion after a number of intermediate stages (see Figure 2). The quantities $\Delta H_{i} / H$ decrease. We shall denote the moment when this begins by $t_{\mathrm{F}}$.

What is the pattern of the further expansion in these models? The models expand almost isotropically according to a law deviating slightly from the Friedmann case $H_{\mathrm{F}}=\frac{1}{2} t^{-1}$. Remember that the equation of state is $p=\varepsilon / 3$.

How small are these deviations? When they have become small enough the quantities $\Delta H_{i} / H$ turn out to attenuate as $\left[\ln \left(t / t_{\mathrm{F}}\right)\right]^{-1}$, i.e. very slowly:

$$
\left(\frac{\Delta H_{i}}{H}\right)_{\max } \approx\left[\ln \left(\frac{t}{t_{\mathrm{F}}}\right)\right]^{-1} \text {. }
$$




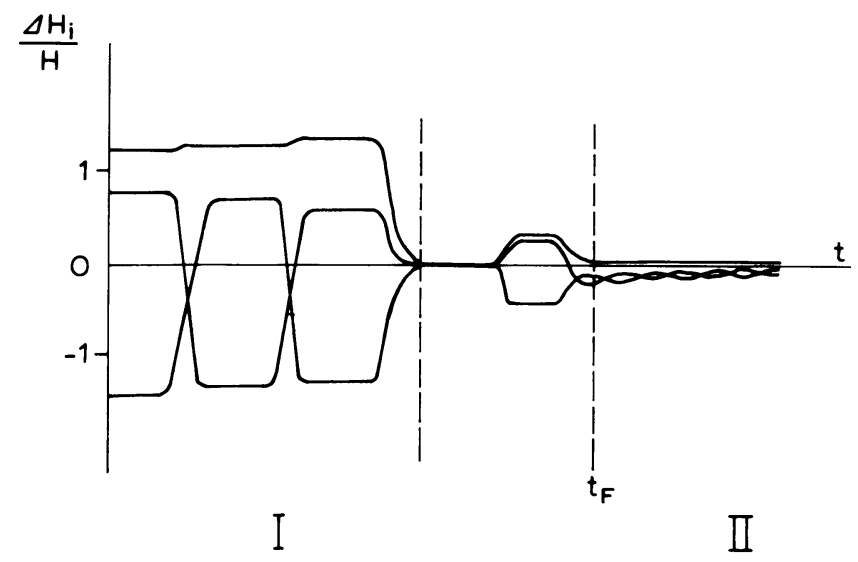

Fig. 2. The behaviour of $\Delta H_{i} / H$ during the expansion of anisotropic cosmological models with anisotropic three-spaces (the types VII and IX).

The residual anisotropy in the curvature is responsible for the extreme slowness of the attenuation of $\Delta H_{i} / H$. Equation (2) is the first important conclusion.

In the late stages of expansion the equation of state changes from $p=\varepsilon / 3$ to $p=0$. Let us denote this moment by $t_{c}$. After $t_{c}$ all deviations from the Friedmannian law attenuate in a power-law manner.

Finally, if the density of matter is not equal to the critical value $\varrho \neq \varrho_{\text {crit }} \equiv 3 H^{2} / 8 \pi G$, there commences a third and final stage of expansion. Up to the late stages of evolution considered above the expansion has proceeded practically as in a Friedmann model with critical density, but now $\varrho$ differs significantly from the critical value $\left(\varrho-\varrho_{\text {crit }}\right) / \varrho \sim 1$. Denote this moment by $t_{M}$. It, like $t_{\mathrm{F}}$ and $t_{c}$, is an arbitrary parameter of the model.

After the moment $t_{M}$ the evolution of models of types VII and IX is entirely different. In the model of type VII, $\varrho<\varrho_{\text {crit }}$ and the three-space is infinite (open model). After $t_{M}$ which we shall call the Milne epoch the anisotropy of deformation $\Delta H_{i} / H$ 'freezes up' in this model (Collins and Hawking (1972) have independently obtained this result):

$$
\frac{\Delta H_{i}}{H}=\text { const. }
$$

The quantities $\Delta H_{i} / H$ are of order of magnitude

$$
\frac{\Delta H_{i}}{H} \equiv x \approx\left(\frac{t_{c}}{t_{M}}\right)^{2 / 3} \frac{8}{8+\ln \left(\frac{t_{c}}{t_{\mathrm{F}}}\right)} .
$$

In one particular case of a model of type VII, $\varrho=\varrho_{c r i t}, t_{M}$ is infinite, and the third stage is absent. 
In a model of the type IX, if the second stage of evolution occurs, $\varrho>\varrho_{\text {crit }}$ and the three-space is closed. In this model after $t_{M}$ the anisotropy of deformation grows rapidly and the model begins to contract as a whole.

Now we will make a couple of remarks about the curvature of the three-dimensional space of these models. Let us begin with models of type VII. The spatial curvature in the models of type VII may be characterized by the three principal values of the metric tensor $\lambda_{1}, \lambda_{2}, \lambda_{3}$ and an arbitrary dimensionless parameter $\alpha$.

Anisotropy of the three-space curvature for models of type VII depends on the relative magnitudes of two dimensionless quantities: $\mu_{1} \equiv\left|\ln \left(\lambda_{1} / \lambda_{2}\right)\right|$ and $\alpha$. If $\sinh$ $\mu_{1} \ll \alpha$, then the curvature is almost isotropic. In the case $\sinh \mu_{1} \gg \alpha$, the curvature is very different in different directions, i.e. there is a strong curvature anisotropy.

Figure 3 shows the evolution of the anisotropy of the spatial curvature for models of Bianchi type VII. You can see that in the first stage the anisotropy is very large and that it oscillates. It damps during the second stage and becomes frozen in the third stage. During the whole period of time $\sinh \mu_{1} \gg \alpha$, i.e. the curvature is always highly anisotropic. However, we shall see that it does not significantly influence the observations.

Let us now turn to the models of type IX. In this case the anisotropy of curvature depends on two quantities $\mu_{1} \equiv\left|\ln \left(\lambda_{1} / \lambda_{2}\right)\right|$ and $\mu_{2} \equiv\left|\ln \left(\lambda_{1} / \lambda_{3}\right)\right|$. The curvature is isotropic if $\mu_{1} \ll 1$ and $\mu_{2} \ll 1$.

Figure 3 shows that $\mu_{2}$ has a moderately high value and the curvature is strongly anisotropic, although $\mu_{1}$ becomes less than 1 in the second stage.

Thus the conclusion about the spatial curvature is this:

In the most general case, assuming that in the course of evolution of models of types VII and IX there exists a long second stage, then the curvature remains strongly anisotropic though the degree of anisotropy decreases in the Friedmann stage. The models of type IX have very small length in one direction and cannot describe the real Universe.

Such is the general expansion pattern of the models. Note that we have considered here the typical case when the parameters of the models $t_{\mathrm{F}}, t_{c}, t_{M}$ satisfy $t_{\mathrm{F}} \ll t_{c} \ll t_{M}$. This is the most important case for applications to the real Universe. The most important features are:

(1) Three stages of expansion: I - vacuum, II - isotropic, III - Milne stage.

(2) An extremely slow decrease of $\Delta H_{i} / H$ in stage II:

$$
\Delta H_{i} / H \sim\left[\ln \left(t / t_{\mathrm{F}}\right)\right]^{-1}
$$

(3) The spatial curvature remains strongly anisotropic in the general case.

Now we come to the basic problem. How are the features listed above connected with observable quantities? What can be said about the initial stage of expansion on the basis of present-day observations? The most sensitive test is measurements of the anisotropy of the background relic radiation. We shall consider only these observations. 


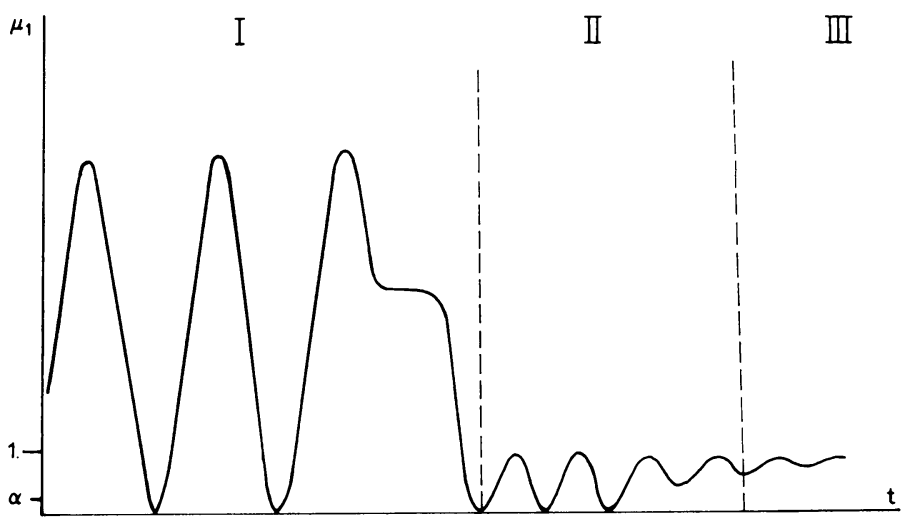

Type IX

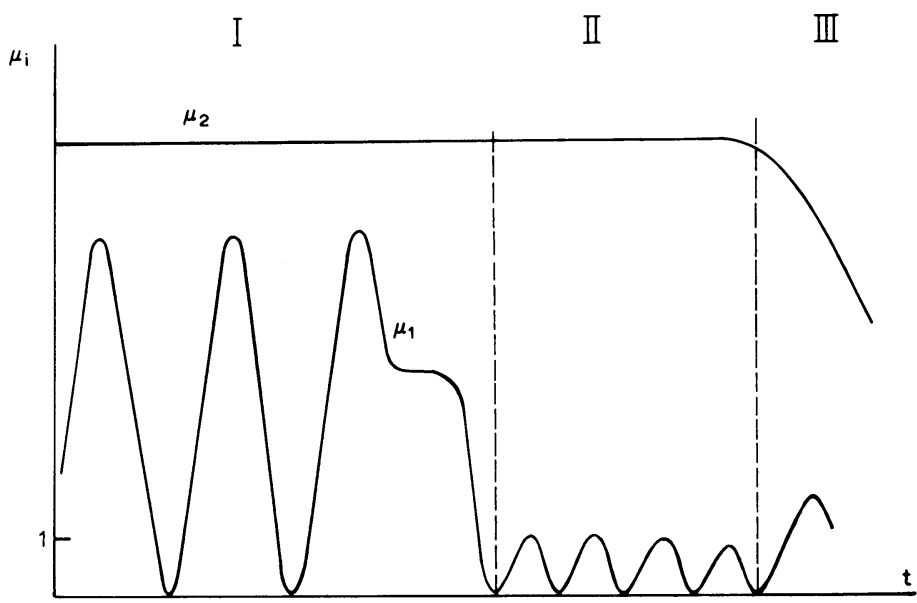

Fig. 3. The behaviour of the curvature anisotropy $\mu_{1}$ and $\mu_{2}$ in the course of the expansion for models of types VII and IX.

The expansion of the Universe today may correspond either to the second stage if we have now $\varrho \approx \varrho_{\text {crit }}$ or to the third stage of models of type VII if $\varrho<\varrho_{\text {crit }}{ }^{*}$

Assume that we are now in the second stage and $\varrho \approx \varrho_{\text {crit }}$. Then the theory predicts anisotropy in the relic radiation. The deviation of the temperature $\Delta T$ from the min-

* Without going into any details notice that the models of type VII may be treated as circularly polarised gravitational waves in spaces of constant curvature (V. N. Lukash, JETP Letters 19, 8 (1974)). 
imal value $T$ should have the following amplitude and distribution over the sky:

$$
\frac{\Delta T}{T} \approx \frac{8}{8+\ln \left(\frac{t_{c}}{t_{\mathrm{F}}}\right)}\left(\frac{t_{c}}{t_{l}}\right)^{2 / 3} \sin ^{2} \theta .
$$

Here $t_{l}$ is the moment when the Universe becomes transparent to the relic radiation (the epoch of recombination). The angle $\theta$ is measured with respect to a particular direction which is also an arbitrary parameter. We adopt $\Delta T=0$ when $\theta=0, \pi$.

Notice that the quantity $\Delta T / T$ has a quadrupole distribution (Figure 4).

Assume next that we are in the third stage and $\varrho<\varrho_{\text {crit }}$. In this case the temperature is distributed over the sky in a different way. There should be a spot on the sky in which $\Delta T / T$ greatly exceeds its value over the rest of the sky. The formation of the spot is connected with the peculiar character of anisotropic expansion in curved space. [Such a spot in the models of Bianchi type $\mathrm{V}$ was discovered by Novikov (1968), and analysed by Grishchuk et al. (1968). For details about the spot in type VII models see Doroshkevich et al. (1973a, b).] We shall adopt $t_{c}<t_{l}<t_{\mathrm{M}}$ as should be the case in the real Universe. The quantity $\Delta T / T$ is $\left(t \gg t_{\mathrm{M}}\right)$ :

$$
\frac{\Delta T}{T}=\frac{z_{l} \mid z_{\mathrm{M}}}{\left(t / t_{\mathrm{M}}\right)^{2}\left(1+\cos \theta \tanh \ln \left(t / t_{\mathrm{M}}\right)\right.}+1 \frac{x \sin ^{2} \theta}{1+\cos \theta \tanh \ln \left(t / t_{\mathrm{M}}\right)} .
$$

Here $z_{l}, z_{\mathrm{M}}$ are the redshifts corresponding to $t_{l}, t_{\mathrm{M}} ; t$ is the present epoch. The distribution pattern is shown in Figure 5. $\Delta T / T$ has a noticeable value in the spot (near $\theta=\pi)$ with angular size $\theta_{1} \approx \varrho / \varrho_{\text {crit }}$. The maximum of $\Delta T / T$ has the same order of magnitude as in the case $\varrho=\varrho_{\text {crit }}$. Note that if $t_{l}>t_{\mathrm{M}}$ then $\Delta T / T \approx x\left[\sin ^{2} \theta /(1+\cos \theta \times\right.$ $\left.\left.\times \tanh \ln \left(t / t_{l}\right)\right)\right] \tanh \ln \left(t / t_{l}\right)$. This distribution has a dipole character over the sky as a whole except for the small spot.

Thus, in both cases (5) and (6) the amplitude of $\Delta T / T$ depends only weakly on the moment of isotropization $t_{\mathrm{F}}$

$$
\left(\frac{\Delta T}{T}\right)_{\max } \approx \frac{8}{8+\ln \left(\frac{t_{c}}{t_{\mathrm{F}}}\right)}\left(\frac{t_{c}}{t_{l}}\right)^{2 / 3} .
$$

This weak dependence is a consequence of the slow decrease of $\Delta H_{i} / H$ during the second stage of evolution. It should be noted that such a slow attenuation of $\Delta H_{i} / H$ when $p=\varepsilon / 3$ may be caused also by weak, directed fluxes of neutrinos or gravitons or by a flux of matter as a whole. Thus, Formula (7) may be regarded as a general one.

Let us compare these results with the observational data. Observations show the large-scale $\Delta T / T$ to be

$$
\frac{\Delta T}{T}<10^{-3}
$$




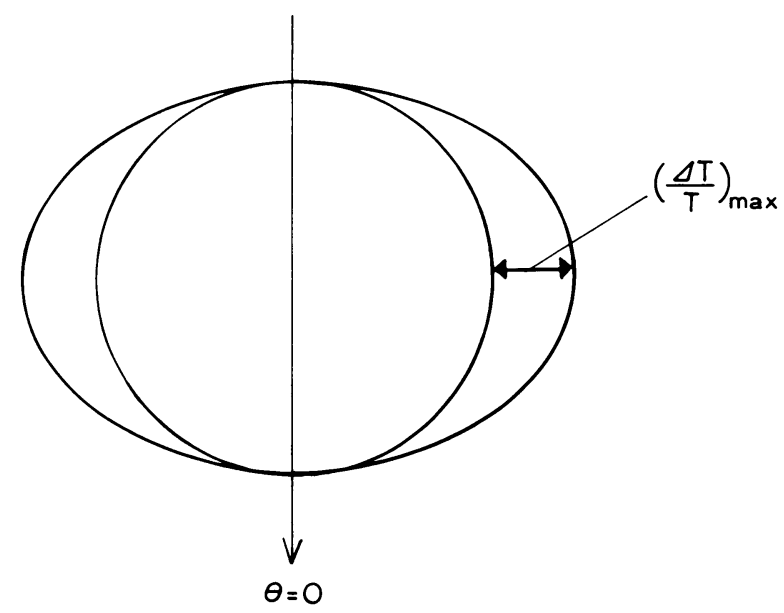

Fig. 4. The angular distribution over the sky of the anisotropy of the relic radiation $\Delta T / T$ in case $\varrho \approx \varrho_{\text {crit }}$.

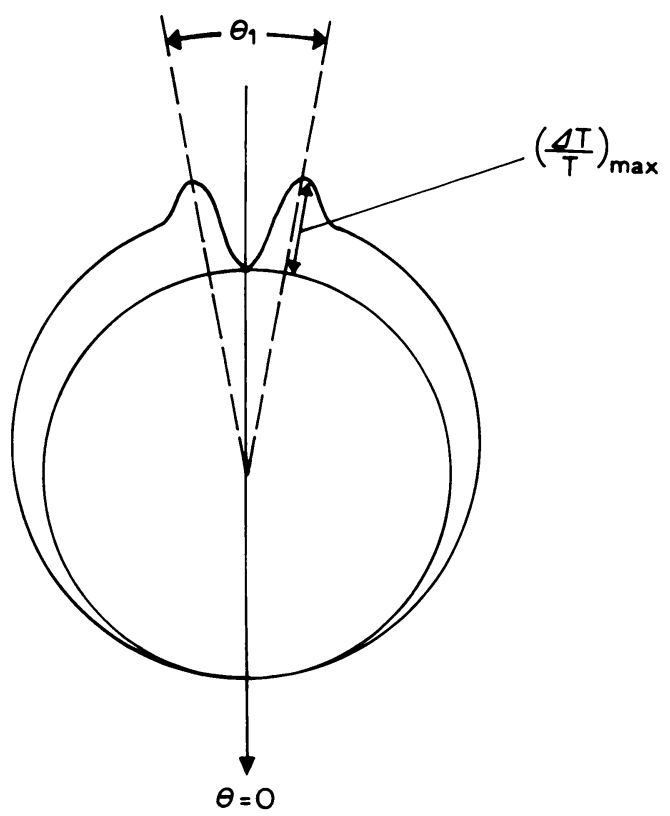

Fig. 5. The angular distribution over the sky of the anisotropy of the relic radiation $\Delta T / T$ in case $\varrho<\varrho_{\text {crit }}$.

Assume first that at the present epoch $\varrho \approx \varrho_{\text {crit }}$ and that Formula $(5)$ is valid. Substituting $\left(t_{c} / t_{l}\right)^{2 / 3} \approx 10^{-1}$ into (5) and using (9) we find that $t_{\mathrm{F}}$ should be close to $t_{\text {Planck }} \approx$ $\approx 10^{-44} \mathrm{~s}$, i.e. to the limit of applicability of non-quantum cosmology. Thus, if $\varrho \approx \varrho_{\text {crit }}$ the observations show that the expansion should have been isotropic from the very beginning.

In the case $\varrho<\varrho_{\text {crit }}$ one cannot draw such a decisive conclusion. $\Delta T / T$ in this case 
is close to its maximum value only in a small spot of angular size $\theta_{1}$. Elsewhere it is much less than $(\Delta T / T)_{\max }$. Since $\Delta T / T$ has not been measured over the whole sky, a small spot with the amplitude $\Delta T / T \sim 0.1$ might have been missed.

\section{Conclusions}

(1) The anisotropy of the relic radiation depends only slightly on the moment of isotropization of the model.

(2) If in accordance with the observations, $\Delta T / T$ is less than $3 \times 10^{-3}$ and the Universe has been transparent to the relic radiation since $z \approx 10^{3}$, the moment of isotropization of the model is extremely early. It is not later than the Planck time $t_{\text {Planck }}=$ $=10^{-43} \mathrm{~s}$.

Thus, the comparison of the theory and the observations leads to a tentative conclusion that the expansion was isotropized very early at $t \approx t_{\mathrm{Planck}} \approx 10^{-43} \mathrm{~s}$.

This conclusion coincides with Prof. Zel'dovich's statement about the quantum creation of pairs by the gravitational field in any anisotropic beginning of the cosmological expansion. His theory leads inevitably to the isotropization of the expansion at $t \approx t_{\text {Planck }}$.

\section{References}

Belinsky, V. A., Lifshitz, E. M., and Khalatnikov, I. M.: 1972, JETP 62, 1606.

Collins, C. B. and Hawking, S. W.: 1972, preprint of Institute of Theoretical Astronomy, Cambridge.

Doroshkevich, A. G., Lukash, V. N., and Novikov, I. D. : 1973a, JETP 63, No. 6.

Doroshkevich, A. G., Lukash, V. N., and Novikov, I. D. : 1973b, preprint of Inst. Appl. Math.

Doroshkevich, A. G., Zel'dovich, Ya. B., and Novikov, I. D.: 1969, Astrofizika 5, 15.

Grishchuk, L. P., Doroshkevich, A. G., and Novikov, I. D. : 1968, JETP 55, 2281.

Heckmann, O. and Schucking, E.: 1962, Gravitation, An Introduction to Current Research, Academic

Press, New York, Ch. 11.

Lukash, V. N.: 1974, Astron. Zh. 51, 281.

Novikov, I. D.: 1968, Astron. Zh. 45, 538.

Novikov, I. D.: 1970, Astron. Zh. 47, 1203.

Schucking, E. and Heckmann, O.: 1958, Conseil Phys., 11 th Solvay, Brussels.

Zel'dovich, Ya. B. and Novikov, I. D. : 1974, Structure and Evolution of the Universe, 'Nauka', Moscow.

\section{DISCUSSION}

Heller: Collins and Hawking have shown that the set of all initial values leading to the isotropization (or Friedmannization, in your language) is of measure zero. Does your picture of cosmic evolution agree with those of Collins and Hawking?

Novikov: I do not agree with some of the Collins-Hawking conclusions. But my definition of isotropisation differs from their's. My definition is connected primarily with the isotropisation of the tensor of deformation. This is one of the reasons for the misunderstanding. We shall discuss these problems after Hawking's lecture. They also discuss degenerate models (in our terminology).

Ozernoy: In making numerical estimates of the isotropization epoch, Dr Novikov assumes that the radiation was last scattered at the redshift of decoupling $\left(z_{e}=10^{3}\right)$. On the other hand, in order to reconcile the measurements of small-scale temperature inhomogeneities observed by Parijskij with any present theory of galaxy formation, the secondary reheating of the intergalactic medium is required and then much smaller values of $z_{e}$, in principle, may be obtained. If the redshift of last scattering was at $z_{e} \approx 8$ (this corresponds to $\Omega=1$ ) the theoretical value $\Delta T / T \leqq 10^{-3}$ that is consistent with the observational con- 
straints, may be obtained. At smaller $\Omega$ the value of $z_{e}$ clearly increases. However, in any reasonable case the isotropization instant, $t_{\mathrm{F}}$, turns out to be much greater than the Planckian value, so that the proximity of $t_{\mathrm{F}}$ to $t_{\mathrm{Plank}} \sim 10^{-43} \mathrm{~s}$ for $z_{e} \approx 10^{3}$ is apparently due to a chance.

Lifshitz: The choice of homogeneous models of types IX and VII is in a certain sense inadequate: type VII does not possess all the necessary properties near the singularity, and type IX is closed and contains as a particular case the closed Friedmann model. It would be more instructive to investigate the type VIII model, which does not contain the closed Friedmann model, and therefore it is difficult to imagine how it could be isotropized.

Novikov: We have investigated models of type VIII as well. I want to emphasise that the properties of isotropisation are the same in all models (types VII, VIII, IX) during the second stage of evolution - the most important stage.

Misner: Is there any possible set of refined observational results which would lead, via your formula, to an indicated isotropization time very much earlier than $10^{-43} \mathrm{~s}$ ?

Novikov: If observations give the result $\Delta T / T \ll$ some value corresponding to $t_{\mathrm{F}} \ll 10^{-43} \mathrm{~s}$, then this means that the Universe expanded isotropically from the very beginning without any anisotropic stage. 